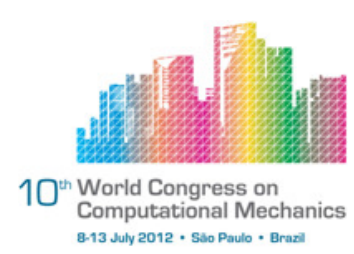

\title{
CAD-BASED MULTI-OBJECTIVE SHAPE OPTIMIZATION METHOD WITH META-PARAMETERS
}

\author{
P. Froment ${ }^{1,2}$, F. Gillot ${ }^{1}$, L. Jezequel ${ }^{1}$, Y. Tourbier ${ }^{2}$ \\ 1 Tribology and System Dynamics Laboratory, Ecole Centrale de Lyon (pierre.froment@ \\ ec-lyon.fr, frederic.gillot@ec-lyon.fr, louis.jezequel@ec-lyon.fr) \\ ${ }^{2}$ Research Department of Optimization and Numerical Simulations, Renault SA, Paris (yves. \\ tourbier@renault.com)
}

\begin{abstract}
Current design loops for shape optimizations allows significant improvements in relation to the functions that need to be optimized, and are widely used in industry. Among these approaches, parametric shape optimization allows rapid enhancement of the shape, on the condition that the design space is confined enough in order to be explored within a reasonable computational time.

This paper introduces a CAD-based large-scale shape optimization method for products requiring significant computational cost, for instance in multiphysics simulations. A two-step scheme is used to achieve the intended results. The first step is to build a base of meta-parameters from an initial learning set. The number of meta-parameters is defined according to the final computational cost that is intended.

These meta-parameters are generated by an artificial neural network pre-trained on a sample set of simulations. The meta-parameters represent a combination of parameters having a similar influence towards the variation of objective functions.

Subsequently, the previous meta-parameters are used to rapidly reach a limited design space close to the optimum. To prove the efficiency and accuracy of the method, the workflow schedule is applied on shape optimization of a car body. The objective is to optimize both the side force coefficient and the yaw moment coefficient, all while taking into account the drag coefficient.
\end{abstract}

Keywords: Shape optimization, Parameterization, Multiphysics.

\section{INTRODUCTION}

Within the framework of mechanical products, designers aim at finding an optimal shape with regard to a set of multiphysics constraints such as aerodynamics, aeroacoustics, thermo-mechanics, aesthetics, etc.

The development process in CFD today is based exclusively using CAD models. Current design loops begin with the drafting of technical specifications, followed by the definition 
of the available volume for the design space. A pre-study is then conducted to define the architecture of the part. The next step is to create the initial geometric model: the working drawing is evaluated through numerical evaluations with regard to each engineering field involved in the study. The analysis of the results identifies the necessary changes to enhance the initial model. In the end, a design that fulfills most requirements is adopted, completing the design loop.

During the research of the optimal shape, this loop is repeated sequentially several times to cover a larger design space. The optimum obtained during this process corresponds to an overall compromise with regards to the requirements. Advanced methods try to use an optimization on the CAD parameters of the design that allows enhancing the shape of the geometry, with the exception that the design space should be confined enough in order to be explored within a reasonable computational time.

To cover a design space as large as possible, i.e. the one offering the most freedom, it is necessary to have an extremely flexible geometry. To achieve this, it is worth multiplying the geometric parameters that define the shape. The multiplication of parameters increases the effort needed to determine the influence that variation of each parameters have on design sensitivity. This limitation makes it very difficult to design a generic CAD model that adheres to a large neighborhood of solutions, all while restricting the number of parameters.

In an industrial context, due to the lack of parameterization approaches, the complexity of large-scale parametric optimizations and the short design lead-time, the roll-out methods in optimization face difficulties.

\section{STATE OF THE ART}

Within the framework of mechanical part design, shape optimization is usually used to reduce design time and provide a reliable and efficient powerful solution to avoid expensive experiments. A wide range of approaches are available to perform such optimizations. According to $[1,2]$, these approaches can be classified into three categories: shape optimization, topology optimization and parametric optimization. Each kind of shape optimization has its pros and cons that are summarized in table 1.

The ability of an optimization method to provide an innovative and efficient design depends on the initial geometric parameterization. Both shape and topologic parameterizations provide a larger design space than parametric optimization (sizing optimization) but this is done to the detriment of the computational resources required. On the other hand, increasing the number of parameters in a geometric optimization (CAD) involves the increase of the design space, which entails an increase in the number of optimization loops.

The parameterization for shape optimization relies on the displacement of the initial grid nodes [3, 4]. Thus, the optimal shape design is given by a mesh deformation of the initial part. This parameterization allows a wide domain of exploration that depends on the initial design, the initial discretization of the part and the allowed range of displacement for the nodes. The underlying idea of topology optimization is to find the optimal density distribution in an initial design space [5]. Since the parameters of this method are the density of each element, the topologic parameterization depends on the initial discretization of the design space. This space has no physical meaning. Parametric optimization (CAD) is based 
on a set of dimensions (radii, lengths...) which allows changes to the geometry thanks to a design table [6].

Table 1. Comparison between different types of shape optimization

\begin{tabular}{cccccc}
\hline $\begin{array}{c}\text { Optimisation } \\
\text { type }\end{array}$ & Parameters & $\begin{array}{c}\text { Number of } \\
\text { parameters }\end{array}$ & $\begin{array}{c}\text { Design } \\
\text { space }\end{array}$ & $\begin{array}{c}\text { Topologic } \\
\text { changes }\end{array}$ & $\begin{array}{c}\text { Manufacturing } \\
\text { process }\end{array}$ \\
\hline Shape & $\begin{array}{c}\text { Nodes } \\
\text { position }\end{array}$ & - & +++ & ++ & + \\
Topology & $\begin{array}{c}\text { Elements } \\
\text { density }\end{array}$ & - & +++ & +++ & - \\
Parametric & $\begin{array}{c}\text { Set of } \\
\text { (CAD) }\end{array}$ & + & + & + & +++ \\
\hline
\end{tabular}

Another important aspect for parameterization is its ability to integrate a given manufacturing process $[7,8,9,4]$. A solution provided by a shape optimization has to be postprocessed in order to meet manufacturing constraints. The post-processing step generally deteriorates the performance of the theoretical solution since it had not been taken into account during parameterization and optimization steps. A solution provided by a parametric optimization is disposed to respect the manufacturing process since it is directly implemented into the geometry parameterization.

The purpose of this paper is to present a framework for shape optimization, which implements a parametric optimization (CAD) without limiting the number of geometric parameters. We propose to master the amount of necessary evaluations by the use of meta-parameters, without degrading the quality of the convergence to an optimal design.

\section{DESCRIPTION OF THE PROPOSED WORKFLOW SCHEDULE}

The proposed method is based on a CAD model, developed from a dead geometry (stemmed from a neutral format such as IGES, STEP). The CAD model meets a hierarchical construction and allows parameterizing rapidly a dead geometry. It includes manufacturing constraints. Parameter ranges are defined according to engineering rules. There is no restriction regarding the number of parameters n. From this model, different configurations are chosen by using design of experiments. An automatic process generates k-geometries.

Secondly, a model of simulation is defined for each engineering field. These models are fully automated and receive the previous k-geometries as inputs. The automation of the computational workflow schedule includes the mesh generation, the physical model, its calculation and the post-processing. This allows making the most of high performance computing: parallel simulations are run automatically for each physics continuum. Thus, a server is exploited to its full potential without any break in continuity. The aim is to enrich a database as early as possible, from which the base of meta-parameters will be built. The latter allow circumventing the difficulties of large-scale optimizations because the number of meta-parameters $\mathrm{m}$ is significantly below the number of geometric parameters $\mathrm{n}$. So, the convergence to the optimum is slightly degraded.

Then, meta-parameters are used in design of experiments. Additional simulations are run, so the Pareto front is hence reached and fully populated. Last, the design team is able 
to choose the Pareto-optimal with regards to requirements, namely the chosen optimal shape cannot improve a performance while deteriorating another one. Figure 1 summarizes the workflow schedule.

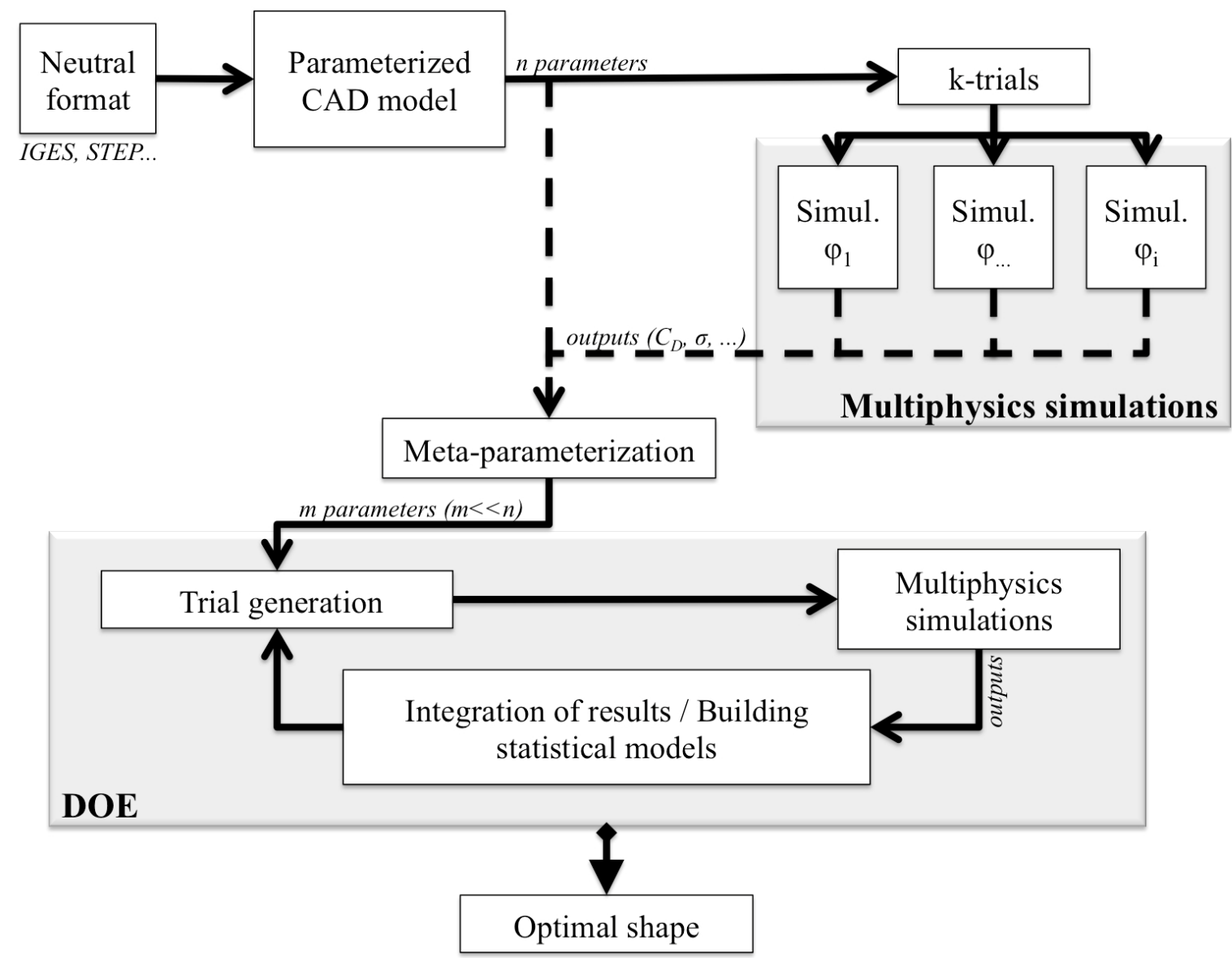

Figure 1. Block diagram of the workflow schedule.

\subsection{Description of an industrial case}

The proposed workflow schedule is applied to an industrial case. We focus on optimizing the external shape of a vehicle with respect to aerodynamic performance. This optimization is particularly time consuming: as a first step, a parametric model that mimics the design spirit needs to be created, i.e. it should respect the rays of light. As a matter of fact, the distinctive feature lines of a vehicle has to be preserved. Only modifications that mimic the design are allowed and these should increase the aerodynamic performance. After an optimization of the shape, we should be able to recognize the original design of the model (shape of the vehicle as envisioned) without any difficulty. Moreover, the CAD model should be stable while generating geometries from a design table; secondly, we need to be able to exploit the number of geometric parameters (hundreds). Influential physical parameters for the performances with headwind conditions are already known and engineering rules were created so that new designs meet these requirements in the early sketches. These engineering rules were correlated during wind tunnel experiments and are used for instance to define the profile of the rear quarter panels. What is more, they incorporate manufacturing constraints. 
To provide more security and reduce the carbon footprint of vehicles, influential parameters with crosswind conditions need to be identified. The objective is to re-use the physical parameters coming from headwind experiments to characterize performances with crosswind conditions, then to optimize the external shape of the vehicle for performances with crosswind conditions without deteriorating ones with headwind conditions. The equations are for headwind conditions the drag coefficient $C_{D}$ (cf. eq. (1)), and in particular the coefficient $S C_{D}$ (cf. eq. (2) ) and for crosswind conditions the side force coefficient $C_{y}$ (cf. eq. (3)) and the yaw moment coefficient $C_{n}$ (cf. eq. (4)) [10].

$$
\begin{gathered}
F_{x}=\frac{1}{2} \cdot \rho \cdot V^{2} \cdot S \cdot C_{x} \\
S C_{D}=S \cdot C_{D} \\
F_{y}=\frac{1}{2} \cdot \rho \cdot V^{2} \cdot S \cdot C_{y} \\
N=\frac{1}{2} \cdot \rho \cdot V^{2} \cdot S \cdot L \cdot C_{n}
\end{gathered}
$$

with:

- $S$ : frontal area

- $L$ : length of vehicle

- $\rho$ : density of the ambient air

- $V:$ road speed

Between each design evolution during the development of a vehicle, the time allotted to evaluate the aerodynamic potential and to propose improvements of the shape is restricted to less than 3 weeks.

\subsection{Development of the method}

\subsubsection{Parameterized geometric model of the vehicle}

The starting point is a file with a neutral format (such as IGES or STEP) to which no specific information is attached. The first step of the method is to create a parameterized model of the body inspired by the engineering rules established with headwind conditions. The model must be as close as possible from the dead geometry in order to be validated by the wind tunnel experiments conducted previously (cf. figure 2). 


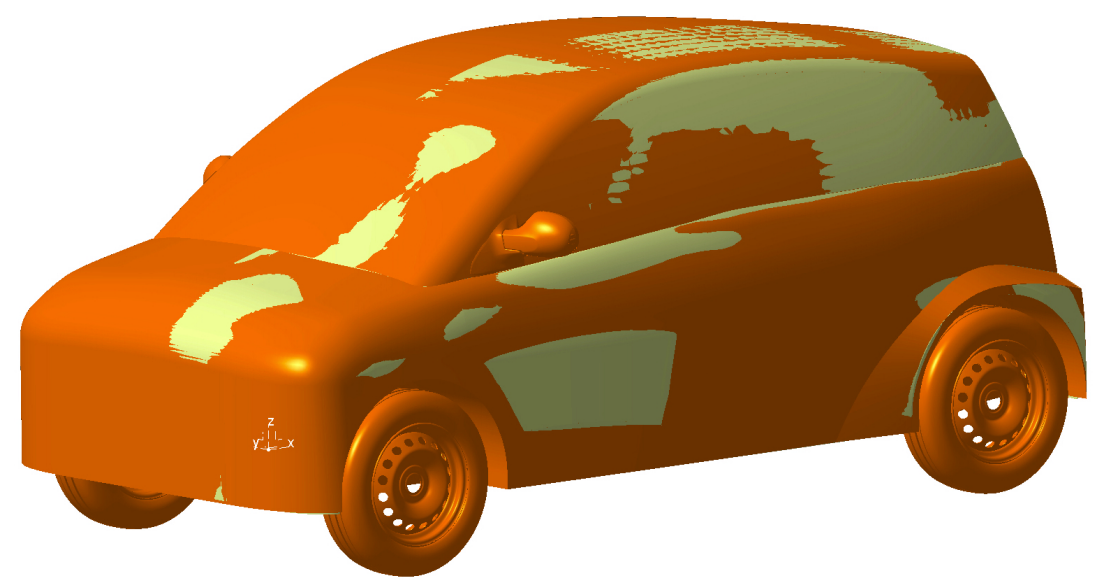

Figure 2. Comparison between the dead geometry (sand color) and the parameterized geometry (orange color).

This model is defined by (100) physical parameters. In order to get the best possible reconstruction rate when generating new geometries, the model adopts a hierarchical and ordered construction that ensures stability while generating geometries from a design table. Initially, all physical parameters related to headwind conditions are integrated. Then, the model is enriched with other parameters in order to ensure both a maximum flexibility and scan a large design space. There is no restriction regarding the number of parameters. The validity of geometries in terms of physical sense is ensured by rules defined on and between parameters: parameter ranges are defined according to engineering rules and manufacturing constraints. Finally, all parameters are listed in a design table with their physical senses (angle of the windshield, wheelbase, overhang...) (cf. figure 3).

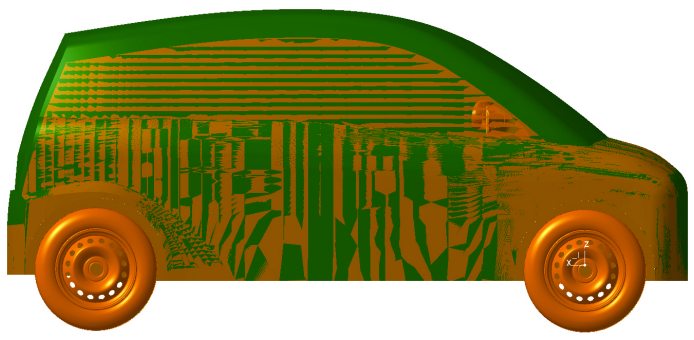

(a) Decrease of the overall height.

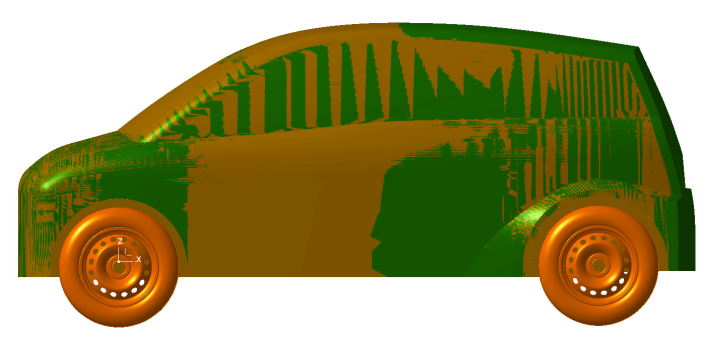

(b) Increase of the rear overhang.

Figure 3. Variation of parameters.

\subsubsection{Geometric model of the wind tunnel}

In order to compare numerical results with experimental results, the flow of the empty wind tunnel was measured to develop a numerical model able to reflect more accurately the real geometry.

The numerical model incorporates the position of the vehicle for crosswind conditions: the vehicle is rotated 20 degrees in the counter-clockwise.

\subsubsection{Simulation model}

This second step aims at building an automated simulation model which takes the geometries of both the wind tunnel and the vehicle as inputs and gives the values of the drag 
coefficient, the yaw moment coefficient, etc. as outputs. For each simulation, only the geometry of the vehicle is re-imported every time.

The geometries generated by the parameterized model revolve in a solution space round the dead geometry. Thus, the dead geometry is taken as reference. The mesh and refinement volumes are defined by this geometry. Refinement volumes must cover all geometries and are positioned to capture all separations of the boundary layer flow that may occur, particularly around the hood, the mirrors, the underbody and the rear (cf. figure 4). Mesh sizes are defined according to the existing engineering rules. A trimmer model is used, mostly made of hexahedral elements, with a prism layer mesher. Finally, the mesh contains 25 million elements.

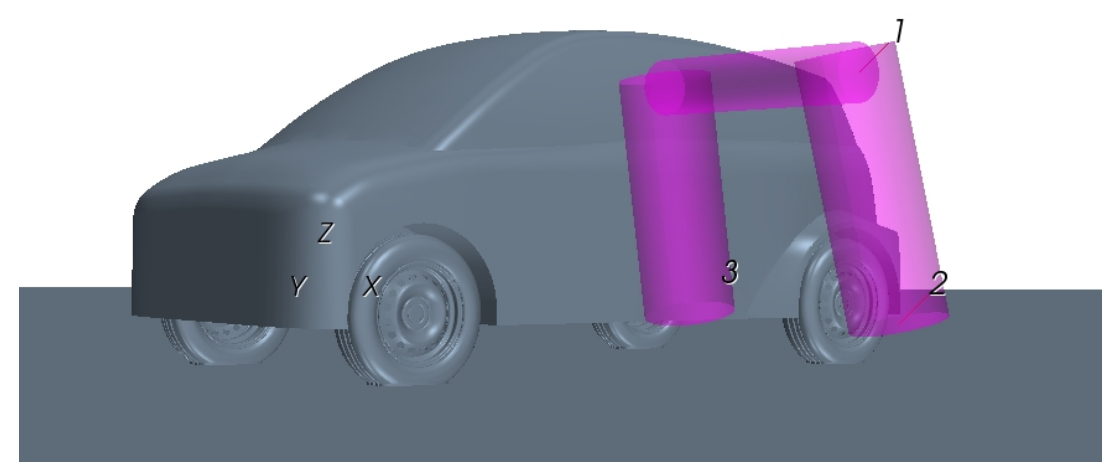

(a) Refinement volumes.

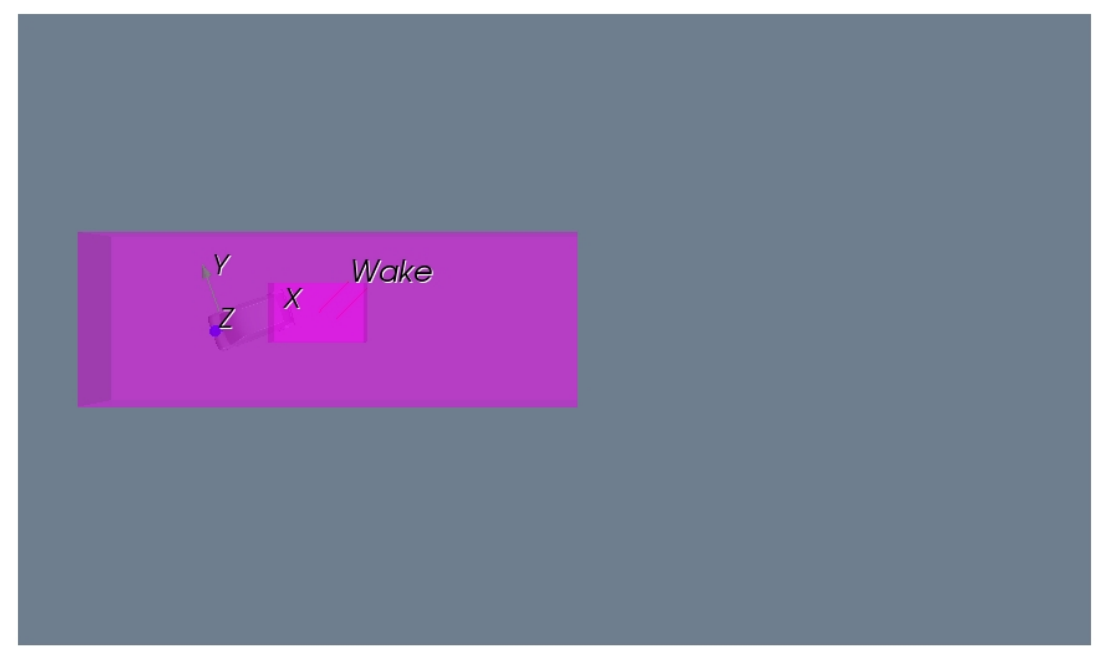

(b) Wake refinement volume.

Figure 4. Position of the refinement volumes.

In order to have the same conditions as the experiments, it is assumed that the ground scrolls and that the air inlets of the vehicle are clogged.

In addition, it is necessary to capture the sensitivity of the measured physical quantities $\left(S C_{D}, C_{y}, C_{n}\right)$ due to a geometric modification. These sensitivities are in the range of some thousandth of $\mathrm{m}^{2}$ for the $S C_{D}$. Thus, the computer code should be enough predicative to validate the variations of the physical quantities. So, we initially use a steady-state RANS method with a K-Omega turbulence model and then an unsteady DES method [11]. 


\subsubsection{Creation of meta-parameters}

The meta-parameterization is achieved by the use of an artificial neural network. Its main properties are the selection of variables. The input vector of the artificial neural network is made of geometric parameters. The network consists of a succession of layers whose number is previously defined by the number of meta-parameters wanted in the end. The number of meta-parameters is directly related to the time that we have for the study. Each layer (i) is composed of $\mathrm{n}_{i}$ neurons, whose inputs are the $\mathrm{n}_{i-1}$ neurons of the preceding layer. The artificial neural network is trained on a learning set, constituted in our case by the k-simulations conducted previously. The last layer corresponds to the meta-parameters [12].

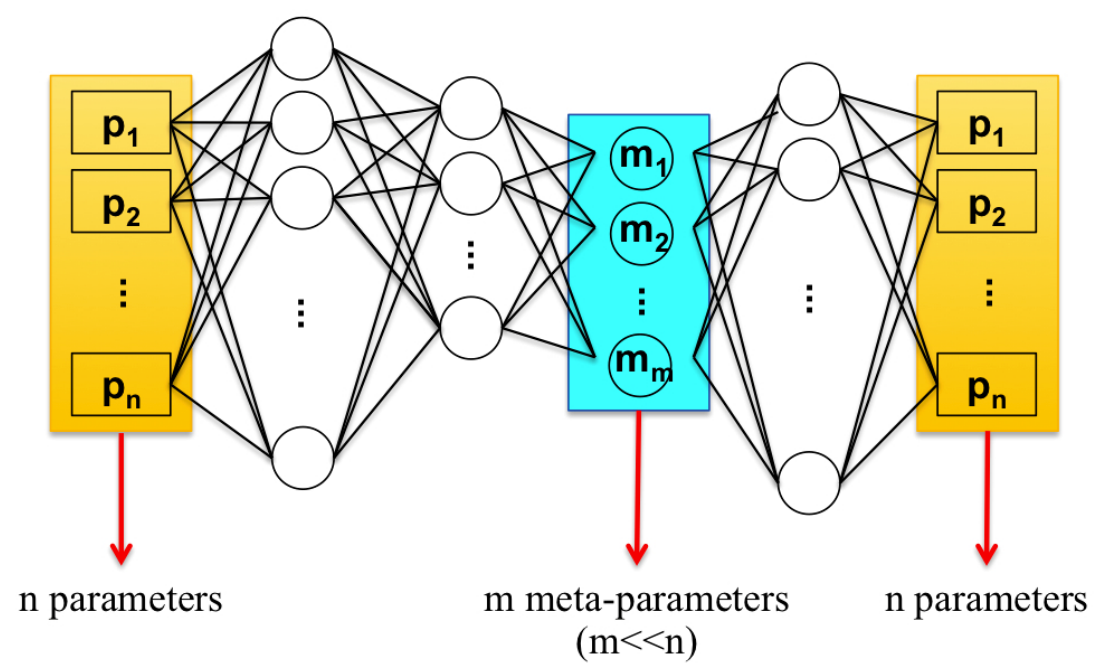

Figure 5. Artificial Neural Network.

Each meta-parameter represents a combination of the geometric parameters whose influence is similar towards the physical simulations $\phi_{i}$. The design space described by the geometric parameters is hence reduced intelligently, browsing an interesting subspace to quickly reach the optimum.

\section{RESULTS DISCUSSION}

\subsection{Number of parameters}

As mentioned earlier, the aim is to design a generic CAD model that adheres to a large neighborhood of solutions, so it should be flexible. The CAD model has about a hundred geometric parameters that characterize the external shape of the vehicle. The number of metaparameters is defined according to the time allotted to deliver the results. The number of layers was set to four so as to keep about $20 \%$ of the number of the initial parameters.

\subsection{Design of experiments to create the learning base}

The issue concerns the choice of the k-geometries for the learning base. Some calculation points should be chosen in a space of $n$ parameters. Hence, a supersaturated design of experiments is used: the criteria chosen for the algorithm is the minimization of the maximum of the absolute value of the correlation coefficient over all possible pairs in a linear model. 


\subsection{Computational time}

The entire optimization loop is automated, in order to make the most of high performance computing resources available. Each simulation is run on 64 cores and takes about thirty hours for completion; ten simulations are run in parallel.

A complete design of experiments would need about $2^{100}$ calculations, which is impossible with current resources. Similarly, a fractional orthogonal plan would be time-consuming compared to the theoretical gains (few thousandths for each performance).

Our method provides the same gains in a fortnight. The number of meta-parameters is adjusted according to the time allotted to the study. With a limited number of meta-parameters, the initial problem is brought back to a usual problem on which conventional methods can be applied (kriging, etc.).

\subsection{Results of simulations}

In order to compare numerical results with experimental results, physical quantities are measured in the same coordinate system as the ones of the wind tunnels. Thus, forces applied on the vehicle are measured in the vehicle coordinate system (indeed, the weighing machine turns simultaneously with the vehicle) and tomographies are taken in the wind tunnel coordinate system. 


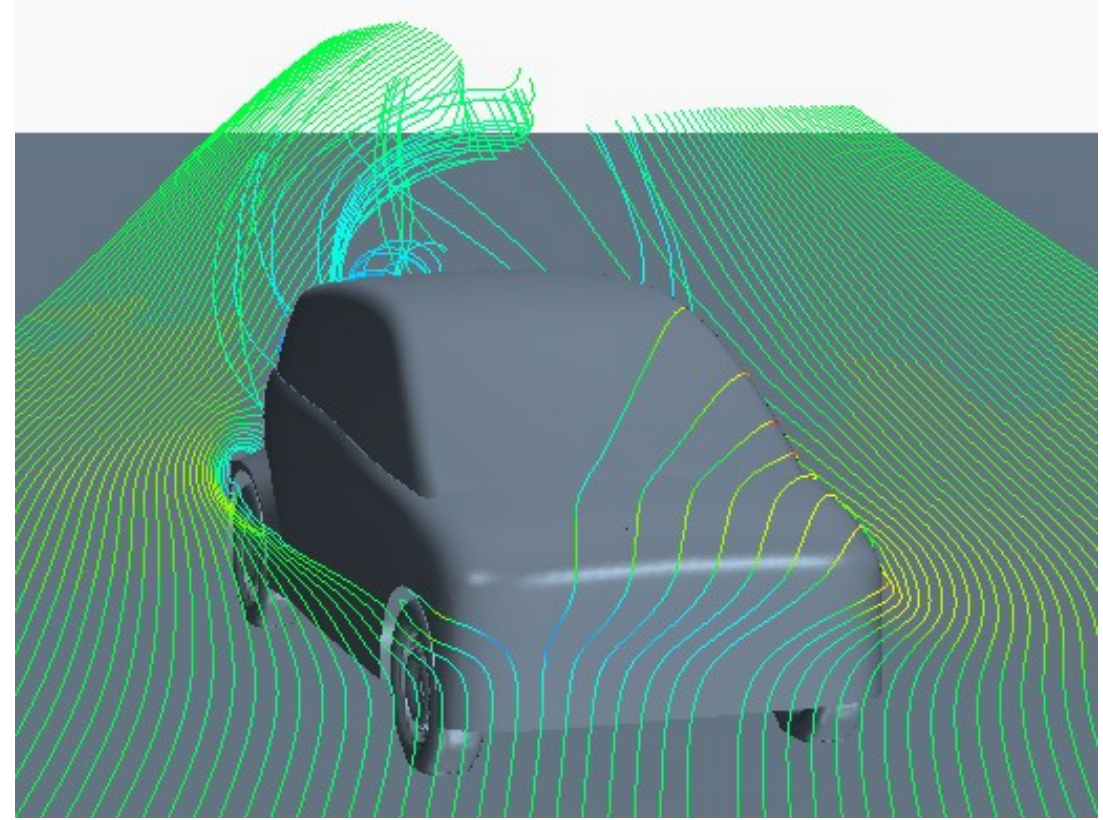

(a) Front view.

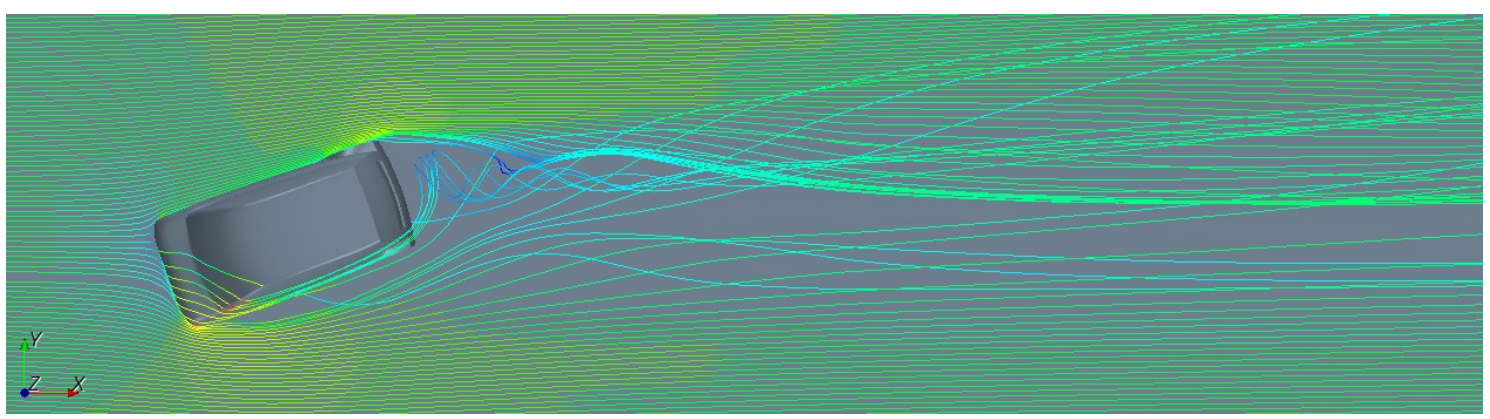

(b) Top view.

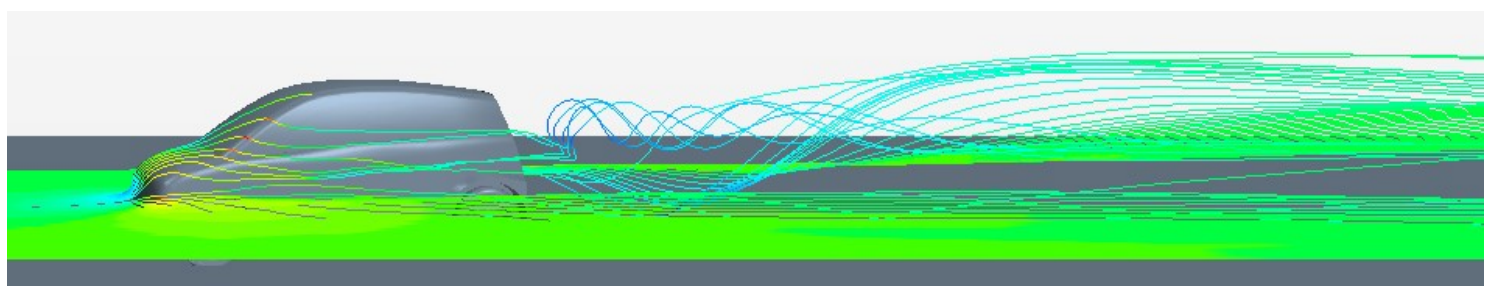

(c) Side view.

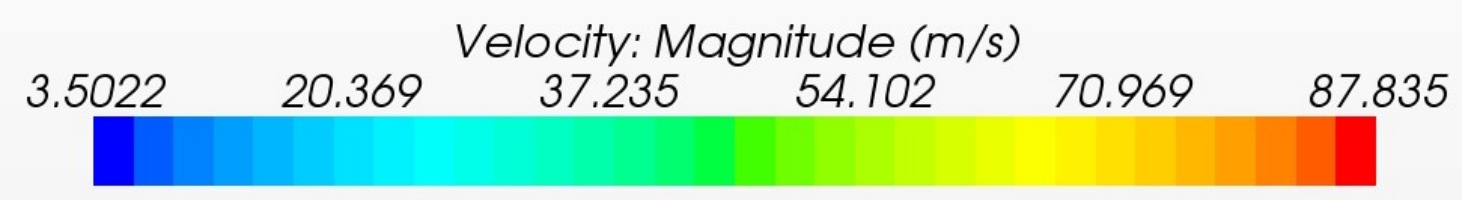

(d) Scale.

Figure 6. Streamlines of the velocity. 


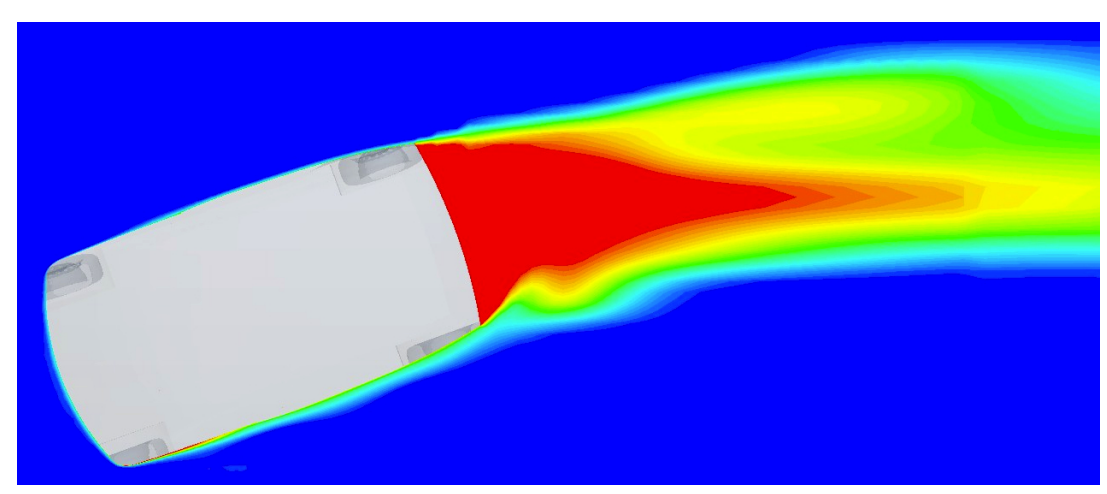

(a) Top view.

$\begin{array}{llllll}0.00000 & 0.24000 & 0.48000 & 0.72000 & 0.96000 & 1.2000\end{array}$

(b) Scale.

Figure 7. Dimensionless pressure coefficient Cp (cf. eq. (5)).

$$
C_{p}=\frac{p-p_{\infty}}{\frac{1}{2} \cdot \rho \cdot V_{\infty}^{2}}
$$

with:

- $p_{\infty}$ : static pressure

- $V_{\infty}$ : free-stream velocity

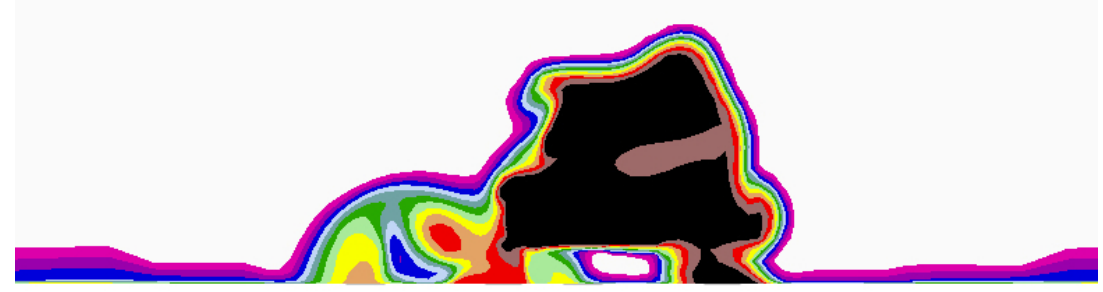

(a) Rear view.

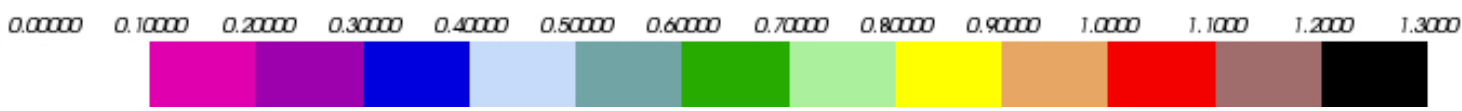

(b) Scale.

Figure 8. Tomography at the rear of the vehicle.

The comparison of results with the experimental data gives an accuracy of the computer code in the range of $2 \%$, that is to say about $0,010 \mathrm{~m}^{2}$ for $S C_{D}$. This difference is reasonable knowing that the repeatability of a same experiment in the wind tunnel is in the range of $\pm 0,003 \mathrm{~m}^{2}$ for the $S C_{D}$.

These results are used to reach and to populate the Pareto front. The optimum within the meaning of Pareto gives a gain for both $S C_{D}$ and $C_{n}$ in the range of some tens of thousandths. 


\section{CONCLUSION}

We have presented in this paper a shape optimization method for products with a short design lead-time in an industrial context. New geometries are generated thanks to a parameterized model, without any restriction regarding the number of parameters that can be used. Then multiphysics simulations are run. Both CAD parameters and simulation results are used in an artificial neural network to create a limited set of meta-parameters. The latter are used in design of experiments. The Pareto front is reached and fully populated, enabling the design team to make better and faster choices with regards to design constraints. This method, breaking away from the usual industrial process, was applied on shape optimization of a car body. The objective was to optimize performances with crosswind conditions without deteriorating performances with headwind conditions. Last, the results were compared with ones obtained by experiments in order to prove the efficiency and accuracy of the method. 


\section{REFERENCES}

[1] First A. A., Second B. B., "Example of nice winter weather". Int. J. Winter Weat. 7, 8-13, 2012.

[1] Saitou A., Izui K., Nishiwaki S., Papalambros P., "A survey of structural optimization in mechanical product development". Journal of Computing and Information Science in Engineering. 5, 214-226, 2005.

[2] Roy R., Hinduja S., Teti R., "Recent advances in engineering design optimization: Challenges and future trend". CIRP Annals - Manufacturing Technology. 57, 697-715, 2008.

[3] Le C., Bruns T., Tortorelli D., "A gradient-based, parameter-free approach to shape optimization". Computer Methods in Applied Mechanics and Engineering. 200, 985-996, 2011.

[4] Kim N. H., Choi K. K., Chen J. S., "Die shape design optimization of sheet metal stamping process using meshfree method". International Journal for Numerical Methods in Engineering. 51, 1385-1405, 2001.

[5] Bendsoe M. P., Sigmund O., "Topology Optimization: Theory, Methods and Applications”. Springer Verlag, Berlin Heidelberg. ISBN 3-540-42992-1, 2003.

[6] Suh M. W., Lee J. H., Cho K. Y., Kim S. I., "Section property method and section shape method for the optimum design of vehicle body structures". International Journal of Vehicle Design. 30, 115-134, 2002.

[7] Harzheim L., Graf G., "Topshape: An attempt to create design proposals including manufacturing constraints”. International Journal of Vehicle Design. 28, 389-409, 2002.

[8] Rais-Rohani M., Solanki K. N., Acar E., Eamon C. D., "Shape and sizing optimization of automotive structures with deterministic and probabilistic design constraints". International Journal of Vehicle Design. 54, 309-338, 2010.

[9] Ponthot J. P., Kleinermann J. P., "Optimization methods for initial/tool shape optimization in metal forming processes". International Journal of Vehicle Design. 39, 14-24, 2005.

[10] Hucho W. H., "Aerodynamics of Road Vehicles". SAE International. ISBN 978-0-76800029-0, 59-97, 1998.

[11] Blazek J., "Computational Fluid Dynamics: principles and applications". Elsevier. ISBN 978-0-08-044506-9, 2005.

[12] Vyshinsky V.V., Dorofeev Y. A., Sviridenko Y. N., "Fast aerodynamic design technologies". 27th International Congress of the Aeronautical Sciences. 2010. 